\title{
Comprehensive comparison of three commercial human whole-exome capture platforms
}

\author{
Asan $^{2,3^{*} \dagger}$, Yu Xu ${ }^{1+}$, Hui Jiang ${ }^{1 \dagger}$, Chris Tyler-Smith ${ }^{4 \dagger}$, Yali Xue ${ }^{4}$, Tao Jiang ${ }^{1}$, Jiawei Wang ${ }^{1}$, Mingzhi Wu ${ }^{1}$, Xiao Liu', \\ Geng Tian', Jun Wang ${ }^{1}$, Jian Wang ${ }^{1}$, Huangming Yang ${ }^{1 *}$ and Xiuqing Zhang ${ }^{1 *}$
}

\begin{abstract}
Background: Exome sequencing, which allows the global analysis of protein coding sequences in the human genome, has become an effective and affordable approach to detecting causative genetic mutations in diseases. Currently, there are several commercial human exome capture platforms; however, the relative performances of these have not been characterized sufficiently to know which is best for a particular study.

Results: We comprehensively compared three platforms: NimbleGen's Sequence Capture Array and SeqCap EZ, and Agilent's SureSelect. We assessed their performance in a variety of ways, including number of genes covered and capture efficacy. Differences that may impact on the choice of platform were that Agilent SureSelect covered approximately 1,100 more genes, while NimbleGen provided better flanking sequence capture. Although all three platforms achieved similar capture specificity of targeted regions, the NimbleGen platforms showed better uniformity of coverage and greater genotype sensitivity at 30- to 100-fold sequencing depth. All three platforms showed similar power in exome SNP calling, including medically relevant SNPs. Compared with genotyping and whole-genome sequencing data, the three platforms achieved a similar accuracy of genotype assignment and SNP detection. Importantly, all three platforms showed similar levels of reproducibility, GC bias and reference allele bias.
\end{abstract}

Conclusions: We demonstrate key differences between the three platforms, particularly advantages of solutions over array capture and the importance of a large gene target set.

\section{Background}

Identifying genetic alterations underlying both rare and common diseases, and also other phenotypic variation, is of particular biological and medical relevance. Even after a decade's effort by the genetics research community since the completion of the first human genome sequences $[1,2]$, most genetic mutations underlying human diseases remain undiscovered. For example, the causative mutations for more than half of human rare diseases [3], the genetic architecture of most common diseases $[4,5]$ and the roles of somatic mutations in most cancers [6] have yet to be characterized. Whole genome re-sequencing can potentially identify these

\footnotetext{
* Correspondence: asan@genomics.org.cn; yanghuangming@genomics.org. cn; zhangxq@genimics.org.cn

† Contributed equally

${ }^{1}$ Beijing Genomics Institute at Shenzhen, 11F, Bei Shan Industrial Zone, Yantian District, Shenzhen 518083, China

${ }^{2}$ Beijing Institute of Genomics, Chinese Academy of Sciences, No.7 Beitucheng West Road, Chaoyang District, Beijing 100029, China Full list of author information is available at the end of the article
}

uncharacterized mutations, and in the past few years great strides have been made in this regard with massively parallel DNA sequencing technologies that can be applied to the whole genome [7-10]. However, the cost of these technologies remains too high for them to be used as a standard method. Recent integration of targeted exome capture with massively parallel sequencing to selectively re-sequence the best-understood functional parts of the human genome - comprising less than $2 \%$ of protein-coding sequences - provides an effective and affordable alternative to identify some of these causative genetic changes.

Several platforms for human exome capture for massively parallel sequencing have been developed and marketed to date [11-14]. In principle, these platforms fall into three classes: DNA-chip-based capture [11,12], DNA-probe-based solution hybridization [14], and RNA-probe-based solution hybridization [13]. These platforms have resulted in great success in pioneering studies hunting for variants causing rare human diseases

\section{Biomed Central}


[11,15-21], and have also been adopted in efforts towards deciphering human common disease and cancer genomes. Yet questions remain about which of these platforms is best for a given application. For example, how many human genes are targeted by each approach and how even is their coverage? How do capture efficacy, technological reproducibility and biases among the different platforms compare? How much input DNA is required and how convenient is each experimentally? How does the cost-effectiveness compare? And what is the power and accuracy of SNP calling, especially for medically important rare SNPs? Up till now, publicly accessible explorations of methodology have been limited to proof-of-concept studies $[11,13,14,22]$, reviews $[23,24]$, or comparisons carried out on only a subset of genes rather than at the whole-genome level [25].

To provide the community with a more solid means to determine the best platform for their experimental needs, we have performed a comprehensive comparison of three commercial human exome capture platforms: NimbleGen's Sequence Capture Array (Human Exome 2.1 M Array, Roche-NimbleGen), NimbleGen's SeqCap EZ (v1.0, Roche-NimbleGen), and Agilent's SureSelect (Human All Exon Kits, Agilent). Each of the three platforms represents one of the classes of exome capture technology currently available. To assess performance with regard to key parameters, including reproducibility, we conducted deep exome capture sequencing for each platform with two technical duplicates $(>30 \times$ and $>60 \times$ coverage) using DNA derived from a cell line from a previously sequenced Asian individual [26]. Other key performance parameters characterized here include the genes targeted, the efficacy of exome capture (including specificity, uniformity and sensitivity), technological biases, and the power and accuracy of exome capture data for subsequent SNP calling. Our findings provide comprehensive insights into the performance of these platforms that will be informative for scientists who use them in searching for human disease genes.

\section{Results}

Human exome capture with the three platforms

We chose platforms that allowed a comparison of the three different methods currently in use for exome capture. The platforms are based on a chip-hybrid method (NimbleGen Sequence Capture Array) or a solutionhybridization method (NimbleGen SeqCap EZ) with a common set of DNA probes, and a solution hybridization method with RNA probes (Agilent SureSelect). The test DNA sample was from a cell line derived from the individual used in the YanHuang whole-genome sequencing analysis [26], allowing comparison with the existing high-coverage genome sequence.
We sought to comprehensively compare the performance of the three exome capture platforms using the best protocols and experimental design for each. We therefore optimized the standard library construction protocols for all three platforms (see Materials and methods): we minimized the input DNA to $10 \mu \mathrm{g}, 3 \mu \mathrm{g}$, and $3 \mu \mathrm{g}$ for Sequence Capture Array, SeqCap EZ and SureSelect, respectively, and set pre-capture PCR to four cycles and post-capture PCR to ten cycles for all three platforms. We included duplicates for each technique to ensure the reliability and assess the reproducibility of data production. We thus constructed a total of six libraries for the three platforms and used the HiSeq2000 to initially produce $>30$-fold coverage of unique mapped paired-end 90-bp reads (PE90) for each library. We further sequenced one of the two replicates for each platform to $>60$-fold coverage to obtain a combined coverage of approximately 100-fold for the purpose of discovering the impact of sequence depth on genotype calling for each of the platforms.

\section{Targeted genes and coverage}

One intrinsic feature of exome capture is its capacity for simultaneous interrogation of multiple targets depending directly on the genes targeted by the capture probes. We first compared the targeted genes and their coverage among the three platforms. As the two platforms (array and EZ) developed by NimbleGen shared a common set of targets, we only needed to compare the Agilent and one NimbleGen platform. We annotated protein-coding genes using a merged dataset of 21,326 genes from the CCDS database (release of 27 March 2009), refGen (release of 21 April 2009) and EnsemblGen databases (release 54), and microRNA genes using 719 genes from the human microRNA database (version 13.0). We also included the 200-bp most-flanking regions from both ends of the targeted sequences: typically, 200-bp flanking regions are co-captured with capture libraries constructed from 200- to 250-bp fragments.

The two target sets were $34.1 \mathrm{Mb}$ (NimbleGen) and $37.6 \mathrm{Mb}$ (Agilent) in size, and shared $30 \mathrm{Mb}$ of targets in common, leaving $4.1 \mathrm{Mb}$ specific to NimbleGen and 7.6 Mb specific to Agilent (Table S1 in Additional file 1). Correspondingly, although both target sets contain similar percentages of functional elements (exomic, $>71 \%$; intronic, $>24 \%$; and others, $<5 \%$ ), Agilent covered approximately 1,000 more protein-coding genes and approximately 100 more microRNA genes $(17,199$ protein coding genes, $80.6 \%$ of the database total; 658 microRNA genes, $91.4 \%)$ than NimbleGen $(16,188$ protein-coding genes, 75.9\%; 550 microRNA genes, 76.5\%) (Table S2 in Additional file 1). Of those protein-coding genes, 15,883 overlapped between NimbleGen and Agilent, while 305 were unique to NimbleGen and 1,316 
were unique to Agilent. Further analyses showed no over-representation of any class of annotated disease genes in the NimbleGen- or Agilent-specific genes (Table S3 in Additional file 1). In addition, both included roughly 1.6 transcripts per gene, a value consistent with the average number of transcripts per gene in the RefSeq database. The results indicated that the majority of known human genes and their splice alternatives were well accounted for in both capture probe designs.

We assessed the coverage of the protein-coding sequences (CDs) by the two platforms, and again, Agilent-targeted regions showed much better coverage (72.0\% of targeted genes with $>95 \%$ CDs, and $78.5 \%$ with $>90 \%$ CDs) than NimbleGen's (46.1\% of targeted genes with $>95 \% \mathrm{CDs}$, and $61.5 \%$ with $>90 \% \mathrm{CDs}$ ) (Figure $\mathrm{S} 1$ in Additional file 2). However, when including the flanking regions, the coverage was much more improved for NimbleGen $(74.2 \%$ targeted genes with $>95 \%$ CDs and $76.0 \%$ with $>90 \%$ CDs) than for Agilent (82.0\% targeted genes with $>95 \%$ CDs and $83.0 \%$ with $>90 \%$ CDs) (Figure S1 in Additional file 2). This reduced the gap in $\mathrm{CD}$ coverage rate (from $>17 \%$ to $<8 \%$ ) between the two analysis sets and indicated a more important role of flanking region capture for NimbleGen.

To obtain more detailed information about the target coverage of these two systems, we looked specifically at their ability to interrogate human disease genes using four known data sets (see below). Of the 5,231 unique genes collected from the Online Mendelian Inheritance in Man database (OMIM; release of 10 March 2011), Human Gene Mutation Database (HGMD; Professional 2009.2), and Genome-Wide Association Study (GWAS; release of 3 March 2011) and Cancer Genome Project (CGP; release of 1 December 2010) databases, Agilent targeted 4,871 with $86 \%$ of genes having $>95 \%$ of CDs covered, in comparison with NimbleGen's 4,642 genes with $83 \%$ of genes and $>95 \%$ of CDs covered (Figure S2 in Additional file 2). Thus, for the current pool of disease genes, both could interrogate most known genes, especially those linked to rare diseases, for which $85 \%$ of known causative mutations occur in CDs. This makes both capture methods especially attractive for rare disease gene identification and analysis.

\section{Exome capture specificity}

To assess the extent of exome enrichment, we compared the capture specificity of the three platforms, which was defined as the proportion of reads mapping to target regions. For the two replicates of each platform, we obtained a total of 26 to 80 million filtered reads (2.2 to $7.2 \mathrm{~Gb}$; Table 1 ), roughly corresponding to $>30$ - and $>60$-fold coverage of the targeted regions. We mapped these reads to the human genome (hg18) using the strategy described in the Materials and methods. Although the overall proportion of filtered reads that could be mapped (78.8 to $86.4 \%$ ) or uniquely mapped (69.2 to $82.8 \%$ ) to the human genome differed between the six replicates, the proportions of reads mapped uniquely to targeted regions were more comparable (54.2 to $58.1 \%$ ) among the three platforms (Table 1 ). We also found the percentages of uniquely mapping reads were further improved (by up to $12 \%$ ) for the two NimbleGen platforms by the inclusion of 200-bp flanking regions in the analyses (for the Agilent platform, this was only $2 \%$ ). Thus, the final percentage of usable reads was $66.6 \%$ for the two NimbleGen platforms but was $<60 \%$ for the Agilent platform. These results indicated that there is a general comparability of capture specificity for targeted regions among the three platforms if the mapping method does not include the flanking region sequences. However, under mapping procedures where researchers do include this information, the NimbleGen platforms perform better.

\section{Uniformity of coverage}

The uniformity of sequence depth over targeted regions determines the genotype sensitivity at any given sequence depth in exome capture. The more uniform

Table 1 Capture specificity of the three human exome capture platforms

\begin{tabular}{|c|c|c|c|c|c|c|c|c|c|c|c|}
\hline \multirow[b]{2}{*}{ Replicate $^{a}$} & \multirow[b]{2}{*}{$\begin{array}{l}\text { Reads } \\
\text { (M) }\end{array}$} & \multicolumn{2}{|c|}{ Filtered } & \multicolumn{2}{|c|}{ Mapped to genome } & \multicolumn{2}{|c|}{$\begin{array}{l}\text { Uniquely mapped to } \\
\text { genome }\end{array}$} & \multicolumn{2}{|c|}{$\begin{array}{c}\text { Uniquely mapped to } \\
\text { TR }\end{array}$} & \multicolumn{2}{|c|}{$\begin{array}{c}\text { Uniquely mapped to } \\
\text { TFR }\end{array}$} \\
\hline & & $\begin{array}{c}\text { Read } \\
\text { length }\end{array}$ & $\begin{array}{c}\text { Bases } \\
(\mathrm{Mb})\end{array}$ & $\begin{array}{l}\text { Percent of } \\
\text { reads }\end{array}$ & $\begin{array}{c}\text { Bases } \\
(\mathrm{Mb})\end{array}$ & $\begin{array}{l}\text { Percent of } \\
\text { reads }\end{array}$ & $\begin{array}{l}\text { Bases } \\
(\mathrm{Mb})\end{array}$ & $\begin{array}{l}\text { Percent of } \\
\text { reads }\end{array}$ & $\begin{array}{l}\text { Bases } \\
(\mathrm{Mb})\end{array}$ & $\begin{array}{l}\text { Percent of } \\
\text { reads }\end{array}$ & $\begin{array}{c}\text { Bases } \\
(\mathrm{MB})\end{array}$ \\
\hline NA_r1 & 37 & PE90 & 3,352 & 85.2 & 2,793 & 81.4 & 2,682 & 53.5 & 1,437 & 63.1 & 2,064 \\
\hline NA_r2 & 58 & PE90 & 5,210 & 79.3 & 4,115 & 76.0 & 3,944 & 56.4 & 2,370 & 67.9 & 3,481 \\
\hline NS_r1 & 31 & PE90 & 2,781 & 86.4 & 2,402 & 82.3 & 2,287 & 54.2 & 1,192 & 67.7 & 1,860 \\
\hline NS_r2 & 80 & PE90 & 7,230 & 85.9 & 6,163 & 82.8 & 5,964 & 55.0 & 3,175 & 67.6 & 4,787 \\
\hline AS_r1 & 26 & PE90 & 2,220 & 84.4 & 1,868 & 74.4 & 1,645 & 58.1 & 1,146 & 60.1 & 1,332 \\
\hline AS_r2 & 66 & PE90 & 5,720 & 78.8 & 4,496 & 69.2 & 3,950 & 54.6 & 2,776 & 56.4 & 3,225 \\
\hline
\end{tabular}

${ }^{\mathrm{a}}$ AS, Agilent solution; NA, NimbleGen array; NS, NimbleGen solution; $r 1$ and $\mathrm{r} 2$ are two replicate experiments for each platform. TFR, targeted and flanking regions; $\mathrm{TR}$, targeted regions. 
the sequencing depth on the targeted region is for a platform, the lower the depth of sequencing that is required to obtain a desired genotype sensitivity. To assess this important quality metric, we selected and analyzed a similar number of reads (approximately 25 million filtered reads, on average approximately 30-fold coverage) from each of the six replicates (Table 2). We found that although all three platforms showed high coverage of their own targeted regions at low sequencing depth ( 98 to $99 \%$ with $>1 \times$ ), the Agilent platform showed more bias towards very low and very high coverage $(21 \%$ with $<10 \times, 20 \%$ with $>50 \times)$ than the two NimbleGen platforms $(<15 \%$ with $<10 \times, 7 \%$ with $>50 \times)$. As a result, the two NimbleGen platforms had 10 to $15 \%$ more targeted regions ( 70 to $74 \%$ ) within $10 \times$ to $50 \times$ coverage than the Agilent platform (59\%). This observation was further supported when we looked at the normalized single base sequencing depth distribution (Figure 1). The curve of the two NimbleGen platforms showed less skew to low and high coverage depths, and more evenness around the mean coverage (approximately $30 \times$ ), than that of the Agilent platform; that is, the NimbleGen Array showed the best evenness. In addition, the two NimbleGen platforms also showed better uniformity of coverage in flanking regions (Table 2 ), which is consistent with their better efficiency of capture seen when including the flanking region sequences (Figure S3 in Additional file 2). Thus, the two NimbleGen platforms had a better overall uniformity of sequencing depth than Agilent, which would be expected to impact the relative genotype sensitivity when considering all targets.

\section{Genotype sensitivity}

Although the coverage of $>99 \%$ of each targeted region of more than one-fold using all data sets an upper boundary for exome capture sensitivity for each replicate, only a proportion of these sites gained high-quality genotype assignments. To characterize this issue, we compared the genotype sensitivity in the $30 \times$ data sets (Figure 2a) using the criterion of $>10$-fold coverage and
Phred-like quality $>30$. In these analyses, all three platforms showed very high genotype sensitivity $(>77 \%)$; but, in comparison, the two NimbleGen platforms showed 6 to $8 \%$ higher (>83\%) genotype sensitivity than the Agilent platform (approximately 77\%), which is consistent with their better uniformity in coverage depth.

To obtain a more comprehensive insight, we further analyzed genotype sensitivity at other sequencing depths (Figure 2b) by randomly sampling from the combined sequencing data of the two replicates for each platform. Overall, the genotype sensitivity improved for all three platforms in a similar way as sequencing depth increased, and reached as high as $>92 \%$ at approximately 100 -fold coverage. The genotype sensitivity of the two NimbleGen platforms was often higher than the Agilent platform at a given sequencing depth. For example, genotype sensitivity was between $72 \%$ and $91 \%$ for the NimbleGen platforms at the usual sequencing depth of 20to 50 -fold, while it was 64 to $85 \%$ for the Agilent platform. Of interest, the curves of the two NimbleGen platforms nearly overlapped when sequence coverage depth was $>30$-fold. This indicates that these two platforms, which share a common set of DNA capture probes, have good inter-comparability.

We also analyzed genotype sensitivity at flanking regions; better NimbleGen results further emphasized the importance of the flanking regions for NimbleGen. From the above, we conclude that all three platforms had high genotype calling sensitivity at $>30$-fold coverage (>77\%), with NimbleGen platforms showing slightly better performance.

\section{Reproducibility}

Technical reproducibility reflects the consistency of performance of each exome capture platform. Using the replicates for each of the three exome capture platforms, we determined the level of reproducibility within each platform. In considering inter-platform comparability as well, our evaluation focused on the set of targets that were shared between all three platforms (totaling 182,259 consensus coding sequences

Table 2 Uniformity of depth by three human exome capture platforms

\begin{tabular}{|c|c|c|c|c|c|c|c|c|c|c|c|c|}
\hline \multirow[b]{2}{*}{ Replicate $^{a}$} & \multicolumn{2}{|c|}{ Filtered } & \multicolumn{2}{|c|}{ Mean coverage $(x)$} & \multicolumn{4}{|c|}{ Coverage depth (percent of bases in TR) } & \multicolumn{4}{|c|}{ Coverage depth (percent of bases in FR) } \\
\hline & Reads (M) & Bases (Mb) & On TR & On FR & $0 \times$ & $1 \times$ to $10 \times$ & $10 \times$ to $50 \times$ & $>50 \times$ & $0 \times$ & $1 \times$ to $10 \times$ & $10 \times$ to $50 x$ & $>50 \times$ \\
\hline NA-r1 & 25.5 & 2,280 & 30.8 & 9.3 & 1.3 & 12.3 & 69.8 & 16.6 & 6.8 & 56.2 & 36.8 & 0.2 \\
\hline NA-r2 & 27.1 & 2,421 & 30.0 & 8.5 & 1.0 & 11.2 & 73.8 & 14.1 & 7.4 & 53.1 & 33.1 & 0.1 \\
\hline NS-r1 & 26.1 & 2,338 & 30.1 & 10.8 & 1.7 & 13.0 & 69.7 & 15.6 & 6.1 & 53.1 & 39.5 & 1.3 \\
\hline NS-r2 & 25.5 & 2,289 & 30.3 & 9.9 & 1.5 & 13.6 & 68.5 & 16.3 & 6.7 & 56.4 & 35.8 & 1.0 \\
\hline AS-r1 & 25.7 & 2,222 & 32.7 & 3.5 & 2.1 & 18.6 & 59.5 & 19.8 & 46.8 & 42.7 & 10.1 & 0.4 \\
\hline AS-r2 & 25.1 & 2,175 & 32.5 & 3.4 & 2.2 & 19.0 & 59.0 & 19.8 & 47.3 & 42.4 & 9.9 & 0.4 \\
\hline
\end{tabular}

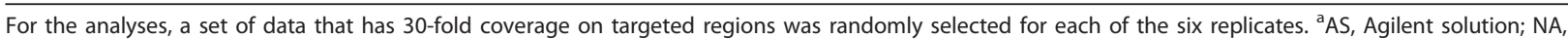
NimbleGen array; NS, NimbleGen solution; $r 1$ and $r 2$ are two replicate experiments for each platform. FR, flanking region; TR, targeted region. 


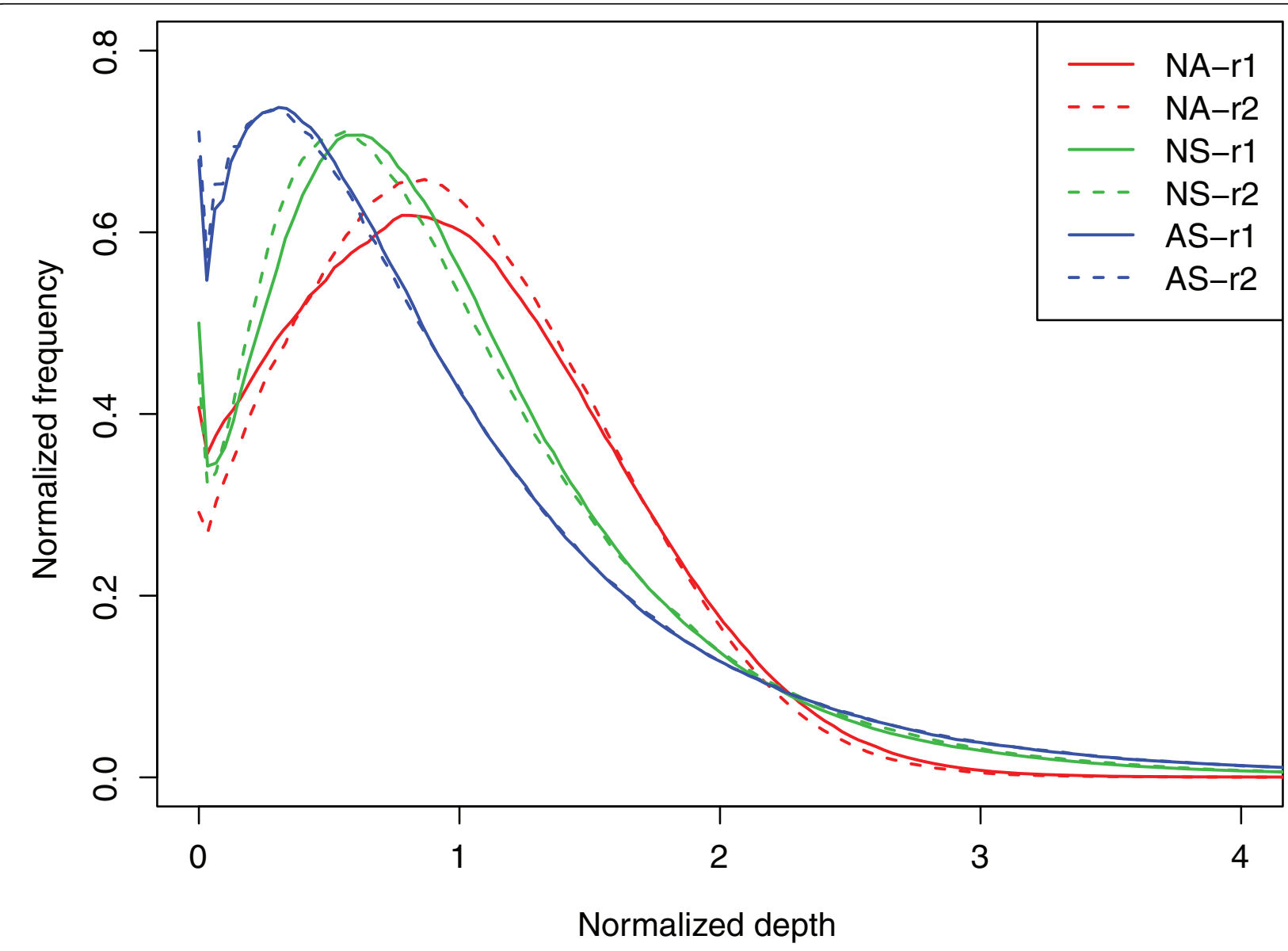

Figure 1 Normalized per-base sequencing-depth distribution on targets. For the purpose of comparison among the three platforms, we selected a set of reads with an average coverage of approximately 30-fold from each replicate. The depth and the frequency (the fraction of a certain depth-level bases for certain sequencing depth-coverage in the total sequencing data) were normalized by the average coverage depth of each replicate on targets. NA-r1 and NA-r2, NS-r1 and NS-r2, and AS-r1 and AS-r2 represent each of two replicates for NimbleGen Sequence Capture Arrays, NimbleGen SeqCap EZ and Agilent SureSelect, respectively.

(CCDSs) covering 25,392,537 bp). This accounted for $70.1 \%$ and $66.1 \%$ of sensitivity in the NimbleGen and Agilent targeted regions, respectively. Using the approximately $30 \times$ data set, we analyzed the correlation of both coverage rate and mean depth on the CCDSs between any two of the six replicates (Figure 3). Each platform showed high intra-platform reproducibility (correlation coefficient at $>0.65$ for coverage rate and $>0.90$ for depth). The lower correlation coefficient for coverage rate $(0.65$ to 0.78$)$ than for mean depth (0.90 to 0.96$)$ was not surprising since the two correlations reflect different aspects of the data - that is, the quantitative sequencing depth and qualitative sequence coverage. For the inter-platform comparison, the two NimbleGen platforms showed higher correlation for both coverage rate and mean depth than the Agilent platform. This is consistent with the fact that the two platforms share a common set of DNA capture probes. These results together indicate generally high and comparable technical reproducibility of the three methods.

\section{GC bias and reference allele bias}

Base composition has been shown to have a systematic effect on capture performance [13]. To explore this effect, we plotted mean sequencing depth against GC content. All three platforms showed biases against extremely low GC content $(<20 \%)$ and high GC content $(>75 \%)$, and the best coverage for GC content of 40 to $60 \%$ (Figure S4 in Additional file 2). However, we also observed a better coverage for the NimbleGen array platform, which had a better coverage of low GC content sequences without reduced coverage of the bestcovered GC content. Thus, extreme GC content still poses a challenge for exome capture, but the chip-hybridization method (NimbleGen array platform) would likely be a better choice for targeted capture of genomic regions with lower GC content. 

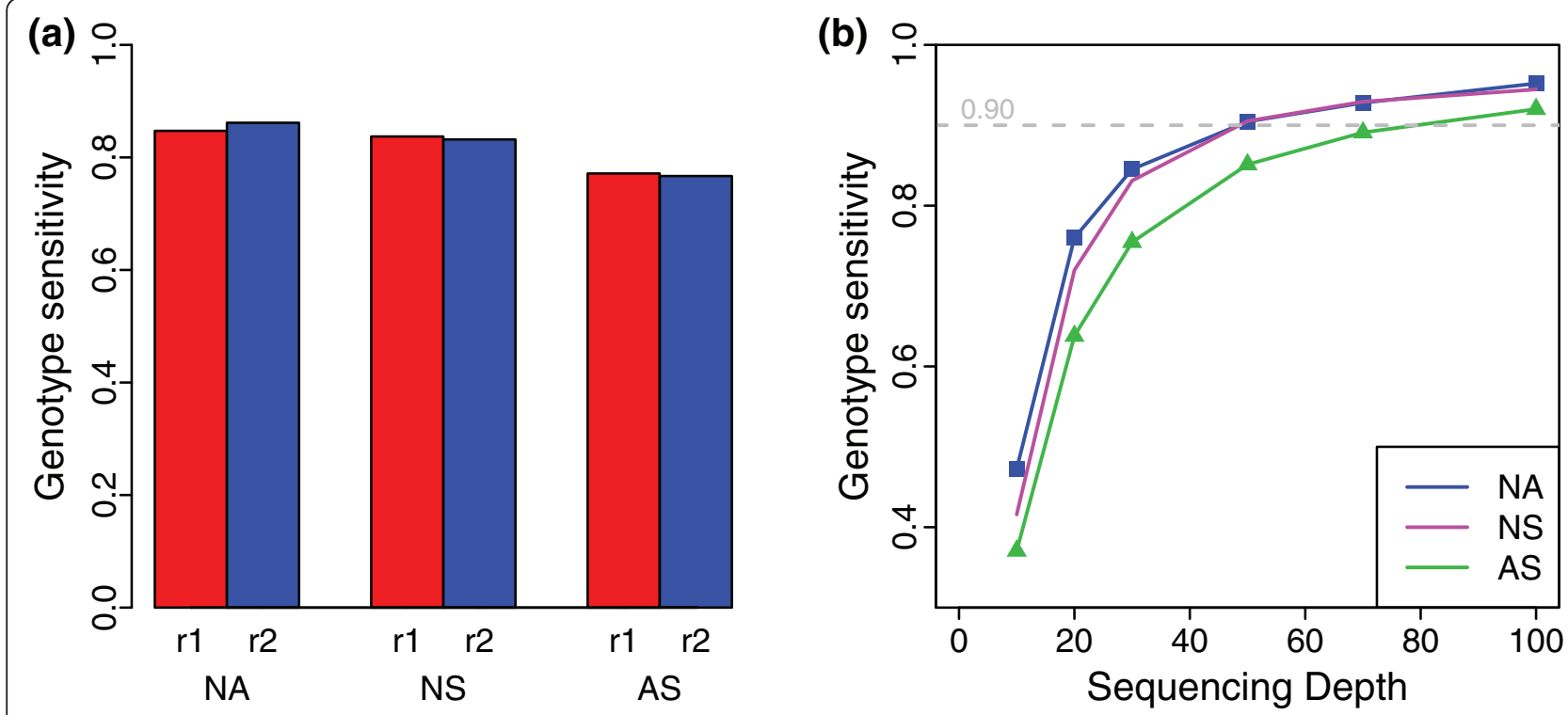

Figure 2 Genotype sensitivity. (a) Genotype sensitivity of six replicates at 30x sequencing depth. (b) Genotype sensitivity as a function of sequencing depth. For the analyses, subsets of reads from two combined replicate datasets for each platform were randomly extracted at different average depths. NA, NS and AS represent NimbleGen Sequence Capture Arrays, NimbleGen SeqCap EZ and Agilent SureSelect, respectively, while $r 1$ and $r 2$ are two replicate experiments for each platform.

The allelic status of the probe sequences could also influence allelic capture efficiency at heterozygous sites, especially in situations where there are a large number of novel alleles being interrogated by exome capture. This occurs because the probes match the reference sequence and might capture perfectly matching library fragments better. To explore the impact of allelic status on the different platforms, we compared the ratio of reference allele depth to total depth for heterozygous sites in each exome capture with that in YanHuang whole-genome shotgun sequencing (WGSS). All three platforms showed consistent and significant biases towards the reference allele in capture (Figure S5 in Additional file 2), whereas WGSS did not have this bias. These results emphasize the need to account for the effect of reference allele bias in exome sequencing of tumors, in which acquired somatic mutations at any frequency may occur.

\section{Non-covered sequences}

Even at 100 -fold sequencing depth, a small proportion of the target region was still not covered by each platform. To gain insight into this issue, we analyzed the base composition of these missed sequences. In total, 97,654 to 190,318 sequences $(0.29$ to $0.56 \%$ of two targeted regions) were not covered at all by the combined full sets of data for each platform. Of these sequences, 19,803 (10 to $20 \%$ of the non-covered sequences) overlapped in all three platforms, and 71,257 (33\% and 70\% of the non-covered sequences) overlapped between the two NimbleGen platforms. The GC content was $>72 \%$ for Agilent, $>80 \%$ for NimbleGen Array, $>79 \%$ for NimbleGen EZ, and $76 \%$ for all shared sequences. Thus, at very high sequencing depth (approximately 100x), the non-covered sequences for all three platforms were biased towards extremely high GC content.

\section{SNP detection}

Given that exome capture is used primarily to identify genetic variants, we compared the SNP detection power among the three platforms. To do so, we called SNPs in the targeted regions together with 200-bp flanking sequence at high quality genotype-assigned sites in each of the approximately $30 \times$ data sets, and annotated them using the combined gene set used in the target annotation. Each platform detected roughly 25,000 to 40,000 SNPs, of which the largest group was from intronic regions, followed by synonymous SNPs and then nonsynonymous SNPs, and finally by other categories (Table S4 in Additional file 1). The over-representation of intronic SNPs was more marked for the two NimbleGen platforms, where it provided over 10,000 more SNPs (35,000 to 40,000 in all) than the Agilent platform $(25,000)$. Given the use of the same DNA and the similar proportion of intronic regions between the NimbleGen and Agilent platforms, this seems to be largely associated with the increased efficiency of capture by the NimbleGen platforms, especially in the flanking sequences. However, for synonymous and non-synonymous SNPs, which together represent the most 


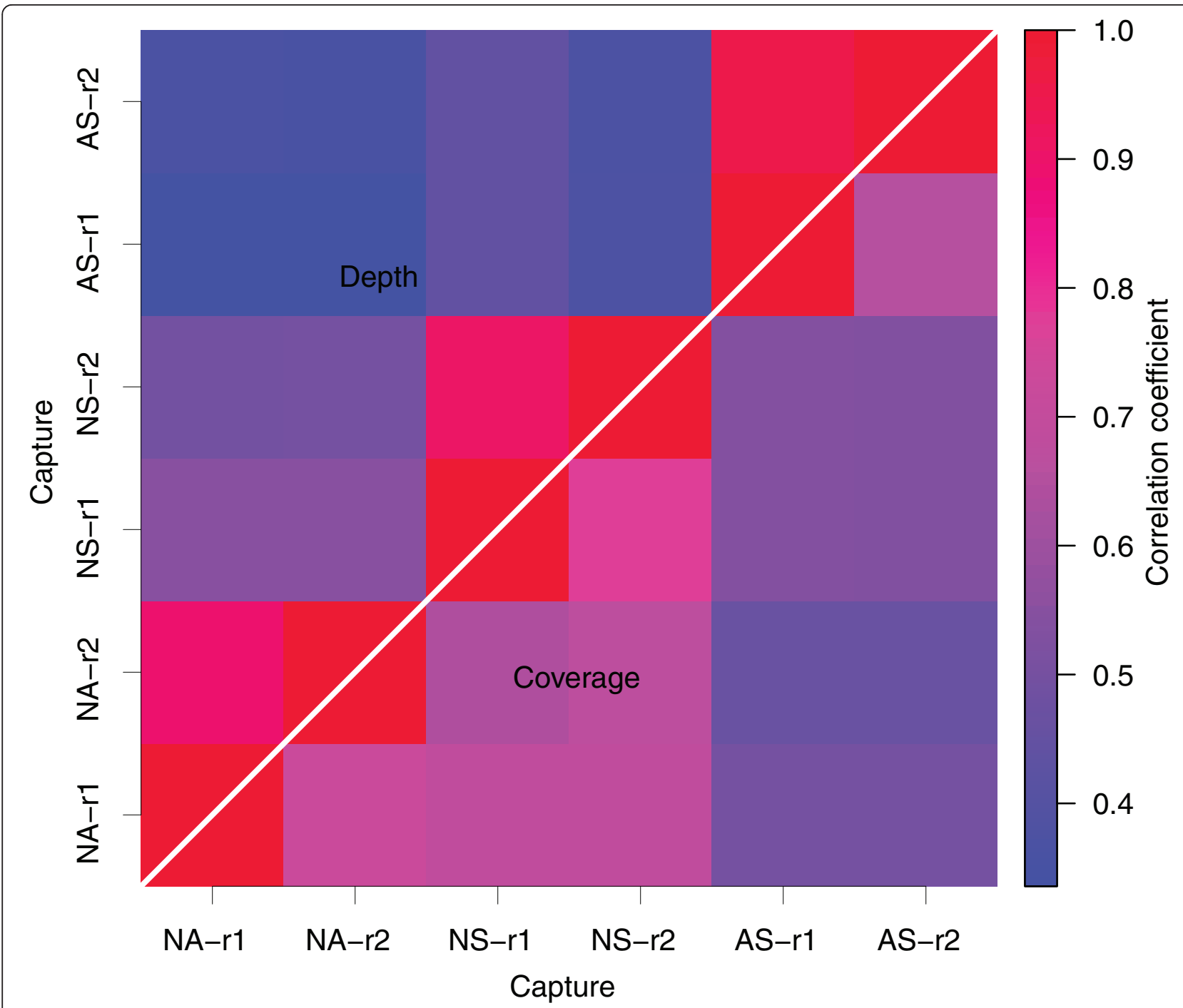

Figure 3 Correlation of sequencing depth and coverage rate on consensus targeted CCDSs. The graph shows pair-wise Pearson correlation coefficients for both sequencing depth (top-left triangle) and coverage rate (bottom-right triangle) based on the 182,259 CCDSs targeted by both Agilent and NimbleGen. NA, NS and AS represent NimbleGen Sequence Capture Arrays, NimbleGen SeqCap EZ and Agilent SureSelect, respectively, while $r 1$ and $r 2$ are two replicate experiments for each platform.

functionally important groups, the Agilent and NimbleGen data showed substantial overlap and nearly similar levels of SNPs per gene to whole genome re-sequencing of the same individual. Thus, the three platforms could interrogate a similar high level of SNPs within proteincoding sequences in their targeted genes, which harbor changes that are most likely to have a functional impact.

\section{Accuracy of genotype and SNP calling}

To assess their accuracy, we compared the genotypes and SNPs from each replicate (30× data) of the three platforms with those from Illumina $1 \mathrm{M}$ beadchip genotyping and WGSS (about 36×) from the YanHuang project [26]. For better data comparability, we also derived genotypes for the WGSS using the same software and criteria as for the exome capture (see Materials and methods).

In comparison with the Illumina $1 \mathrm{M}$ beadchip genotyping, which includes 1,040,000 successfully typed sites, each replicate showed approximately 39,000 to approximately 51,000 overlapping sites depending on the platform, and showed an overall genotype concordance of $>99.81 \%$ for these sites (Table 3 ). In addition, each platform also achieved a similar high concordance rate with those variant sites found by chip genotyping, with $>99.51 \%$ for all the SNP sites, and $>99.56 \%$ for nonreference homozygous sites, and of particular note, even $>99.48 \%$ for heterozygous sites, the genotypes of which 
Table 3 Concordance of genotypes and SNPs

\begin{tabular}{|c|c|c|c|c|c|c|c|c|c|c|c|c|c|c|}
\hline \multirow[b]{2}{*}{ Replicate $^{a}$} & \multicolumn{7}{|c|}{ Concordance with $1 \mathrm{M}$ bead genotyping data } & \multicolumn{7}{|c|}{ Concordance with WGSS data } \\
\hline & $\begin{array}{c}\text { All } \\
\text { genotypes }\end{array}$ & $\begin{array}{c}\text { SNPs } \\
\text { in } 1 \\
M \\
\text { chip }\end{array}$ & $\begin{array}{l}\text { SNPs in } \\
\text { exome } \\
\text { capture }\end{array}$ & $\begin{array}{l}\text { Homs } \\
\text { in } 1 \mathrm{M} \\
\text { chip }\end{array}$ & $\begin{array}{l}\text { Homs } \\
\text { in } \\
\text { exome } \\
\text { capture }\end{array}$ & $\begin{array}{c}\text { Hets } \\
\text { in } 1 \\
M \\
\text { chip }\end{array}$ & $\begin{array}{l}\text { Hets in } \\
\text { exome } \\
\text { capture }\end{array}$ & $\begin{array}{c}\text { All } \\
\text { genotypes }\end{array}$ & $\begin{array}{c}\text { SNPS } \\
\text { in } \\
\text { WGSS }\end{array}$ & $\begin{array}{l}\text { SNPs in } \\
\text { exome } \\
\text { capture }\end{array}$ & $\begin{array}{c}\text { Homs } \\
\text { in } \\
\text { WGSS }\end{array}$ & $\begin{array}{l}\text { Homs } \\
\text { in } \\
\text { exome } \\
\text { capture }\end{array}$ & $\begin{array}{c}\text { Hets } \\
\text { in } \\
\text { WGSS }\end{array}$ & $\begin{array}{l}\text { Hets in } \\
\text { exome } \\
\text { capture }\end{array}$ \\
\hline NA-r1 & 99.846 & 99.641 & 99.826 & 99.649 & 99.987 & 99.633 & 99.687 & 99.999 & 99.216 & 98.636 & 99.951 & 99.868 & 98.683 & 97.750 \\
\hline NA-r2 & 99.854 & 99.670 & 99.835 & 99.708 & 99.975 & 99.637 & 99.714 & 99.999 & 99.264 & 98.616 & 99.943 & 99.850 & 98.768 & 97.724 \\
\hline NS-r1 & 99.854 & 99.679 & 99.819 & 99.682 & 99.951 & 99.676 & 99.707 & 99.998 & 99.211 & 98.396 & 99.974 & 99.747 & 98.657 & 97.426 \\
\hline NS-r2 & 99.849 & 99.660 & 99.841 & 99.684 & 99.987 & 99.640 & 99.716 & 99.999 & 99.197 & 98.706 & 99.979 & 99.752 & 98.629 & 97.949 \\
\hline AS-r1 & 99.816 & 99.526 & 99.823 & 99.571 & 99.948 & 99.486 & 99.712 & 99.998 & 98.783 & 98.021 & 99.917 & 99.824 & 97.945 & 96.703 \\
\hline AS-r2 & 99.815 & 99.514 & 99.805 & 99.556 & 99.880 & 99.477 & 99.738 & 99.998 & 98.762 & 97.972 & 99.927 & 99.771 & 97.893 & 96.645 \\
\hline
\end{tabular}

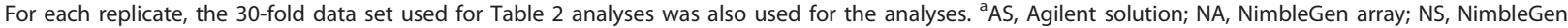
solution; $r 1$ and $r 2$ are two replicate experiments for each platform. Hets, heterozygotes; Homs, homozygotes.

are more difficult to assign than homozygous sites (Table 3). Relatively, the concordance of chip genotyping to the variant sites in each exome capture was also high, with $>99.81 \%$ for all the SNP sites, and $>99.88 \%$ for non-reference homozygous sites, and $>99.71 \%$ for heterozygous sites (Table 3). These comparisons give a maximum estimate of both the false negative rate and false positive rate of $<0.52 \%$ for the three exome captures.

In contrast, the two NimbleGen and Agilent datasets overlapped at $48,000,000$ sites (with $83.8 \%$ sensitivity in targets) and 34,500,000 sites (with $76.2 \%$ sensitivity in targets) with WGSS genotypes, respectively. The substantially higher overlap of NimbleGen was attributed to its greater intronic content. This time, each exome capture platform showed a concordance of $>99.999 \%$ for all overlapping sites, but $>99.20 \%$ for all SNP sites, $>99.92 \%$ for the homozygous non-reference sites and $>97.90 \%$ for the heterozygous sites found in WGSS (Table 3). In comparison, the relative concordance of WGSS to the variant sites called in each exome capture was $>97.97 \%$ for all SNP sites, $>99.75 \%$ for the homozygous nonreference sites, and in particular was reduced to $>96.65 \%$ for the heterozygous sites (Table 3), which is still acceptable. Note that for the heterozygous sites, compared to NimbleGen, Agilent showed approximately $1 \%$ reduction in concordance. In these analyses, cell-line DNA (approximately 40 generations) derived from lymphoblasts was sequenced using a read length of $90 \mathrm{bp}$, while for WGSS reads of $36 \mathrm{bp}$ in length were generated from whole blood DNA. Thus, cell-line mutations, and errors due to increased sequencing length (errors accumulate with sequencing length) in the study may account for part of the decrease in concordance. Based on these results, the general false positive and false negative rate of each exome capture platform for SNP detection was $<3.4 \%$ and $<1.0 \%$, respectively.

Taken together, these results indicate that although slight differences could be observed, accuracy was both high and comparable among the three platforms.

\section{Detection of medically interesting rare mutations}

To further explore the power of the three exome capture platforms at identifying disease-causing rare mutations, we modeled the performance of each with the SNP set present in HGMD (Professional 2009.2) but absent from the 1000 Genomes Project database (BGI in-house data) (Table 4). Of the 39,906 mutations representing 1,931 diseases genes, both Agilent and NimbleGen targeted $>95.8 \%$ sites, and showed $>93.4 \%$ sites with at least $1 \times$ coverage and genotype sensitivity of $>79 \%$ sites $(>10 \times$ coverage and $>$ Q30) at $30 \times$ sequencing depth. But in comparison, Agilent targeted more sites (98.5\% compared to $95.8 \%$ ), and correspondingly showed approximately $1.5 \%$ more covered sites $(>1 \times$ coverage; $95.1 \%$ compared to $93.4 \%$ ) than NimbleGen. In contrast, NimbleGen (the best performance was with NimbleGen Array Capture) showed 1.4\% more genotype sensitivity (80.4\% compared to $79 \%)$, and $3.6 \%$ less low quality coverage sites or uncovered sites $(15.2 \%$ compared to 18.8\%) than Agilent. The number of known potentially disease-causing SNPs detected ranged from 14 to 19 (Table 3). These observations are consistent with the larger targeted gene set of Agilent, and the higher capture efficiency of NimbleGen. Thus, the analyses demonstrated the very high power of the three exome capture platforms for identifying medically interesting rare mutations.

\section{Performance on common targeted regions}

Hitherto, most of the comparisons have been based directly on the current versions of the three platforms, which may not reflect only the intrinsic differences in performance among the three methods, but also the differences in content. To address this issue, we compared key performance parameters on the approximately 30 $\mathrm{Mb}$ of targeted regions in common $(83.3 \mathrm{Mb}$ with flanking sequences; Table S1 in Additional file 1). For specificity, we found that each replicate of the three platforms showed a somewhat reduced unique mapping rate of 
Table 4 Power for identifying disease-causing rare mutations

\begin{tabular}{lcccccc}
\hline & NA-r1 & NA-r2 & NS-r1 & NS-r2 & AS-r1 & AS-r2 \\
\hline High quality genotype assigned sites & 32,139 & 32,674 & 31,750 & 31,923 & 31,685 & 31,353 \\
Reference genotypes & 32,124 & 32,658 & 31,732 & 31,909 & 31,666 & 31,335 \\
SNPs & 15 & 16 & 18 & 14 & 19 & 18 \\
Low quality genotype assigned sites & 6,064 & 5,529 & 6,453 & 6,280 & 7,349 & 7,681 \\
Uncovered & 1,703 & 1,703 & 1,703 & 1,703 & 872 & 872 \\
\hline
\end{tabular}

For each replicate, the 30-fold data set used for Tables 2 and 3 analyses was also used for this analysis. AS, Agilent solution; NA, NimbleGen array; NS, NimbleGen solution; $r 1$ and $r 2$ are two replicate experiments for each platform.

$>44 \%$ filtered reads to the common targeted regions, and that the two NimbleGen platforms achieved, on average, a $12 \%$ higher unique mapping rate than the Agilent platform when including the 200-bp flanking sequences in the analyses (Table S5 Additional file 1). This result is consistent with the initial analyses above.

For uniformity and sensitivity, we also found that each platform showed very similar performance to that above, and that the two NimbleGen platforms performed better than the Agilent one (Table S5 in Additional file 1). For example, at a sequencing depth of 30x, NimbleGen had, on average, approximately $6 \%$ higher genotype sensitivity than Agilent (85\% compared to 79\%). For SNP detection, the detection level of each SNP category in each platform, including the greater detection of intronic SNPs (and thus the total SNP number) by the NimbleGen platforms ( $>13,000$ more SNPs than Agilent, $>35,000$ compared to approximately 22,000), was also similar to the analyses above (Table S4 in Additional file 1); but in comparison, despite general inter-comparability, the two NimbleGen platforms detected approximately 400 more coding SNPs $(12,400$ compared to 12,000$)$ in the common targeted regions while the Agilent platform detected approximately 900 more coding SNPs elsewhere (13,500 compared to 12,600) (Table S4 in Additional file 1). This difference could be explained by the fact that NimbleGen had a better capture efficiency while Agilent targeted an approximately 4-Mb larger region and correspondingly 1,000 more genes.

Finally, for the accuracy of SNP detection and genotypes, we also observed similar false positive and false negative rates for each platform at $30 \times$ coverage (Table S6 in Additional file 1) to that in whole dataset in comparison with the data from array genotyping and WGSS. Thus, we conclude that each platform was highly consistent in performance in the common targeted region analyses here compared with the analyses of the entire content above, which is not surprising given the high overlap (Agilent, $30 \mathrm{Mb} / 34.1 \mathrm{Mb} \approx 80 \%$; NimbleGen, 30 $\mathrm{Mb} / 40 \mathrm{Mb} \approx 88 \%)$.

\section{Discussion}

In this study, we present a comprehensive comparison of three widely adopted human whole-exome capture platforms from two manufacturers. Since the three platforms, in principle, represent the three classes of exome capture technologies currently available, data on their performances likely also reflect the intrinsic power and limitations of exome capture as a technology.

For the current versions of the three platforms, the number of targeted genes and their $\mathrm{CD}$ coverage rate are important considerations for human genetic studies. Although most well-annotated human genes (>76\%) were targeted by all three platforms, Agilent sought to target a larger set of genes (approximately 1,000 more protein-coding genes and approximately 100 more microRNA genes) and thus provided a better coverage of protein-coding sequences. In contrast, NimbleGen emphasized a more important role for flanking regions in capture probe design, and, in practice, had a greater number of genes with a high rate of CD coverage (Figure S6 in Additional file 2) due to better capture efficiency.

Exome capture efficiency is another important factor for comparison of capture platforms. In our hands, we observed that the two NimbleGen platforms showed better capture efficiency than the Agilent platform. Specifically, the two NimbleGen platforms showed approximately $10 \%$ higher capture specificity with the expanded targeted regions (66.6\% compared to $58.3 \%$ ), better uniformity of coverage, and 3 to $7 \%$ more sensitivity in genotype assignment (83 to $95 \%$ compared to 76 to $92 \%$ over the range $30 \times$ to $100 \times$ coverage of targeted regions). Thus, a lower sequencing depth was required for NimbleGen platforms for a given genotype sensitivity on targeted regions, which can impact experimental cost.

The ability to identify SNPs in protein-coding sequences, especially those medically interesting rare mutations, which ultimately measures the power of exome sequencing, was another important consideration. Despite general inter-comparability $(12,500$ to 13,500 SNPs), we found that, at the same sequencing depth (30x), NimbleGen detected a more complete set of SNPs (approximately 400 more SNPs) than Agilent for the common targeted coding sequences due to better exome capture efficiency, but the Agilent platform could 
detect more SNPs (approximately 900 SNPs) in total number due to its larger number of targeted genes. Similarly, for identifying medically interesting rare mutations, we found in model analyses that all three platforms not only showed similar high power at $30 \times$ sequencing depth in interrogating known HGMD mutations filtered to remove 1000 Genomes Project variants present in the general population, but the small differences reflected the general features of each platform (Agilent could target 1.8\% more, and cover 1.5\% more mutation sites, but NimbleGen showed 1.4\% more mutations with high quality genotype assignment).

Input DNA amount, the convenience of conducting experiments and the cost of reagents will also be important considerations. Especially, the amount of DNA required for each method itself will impact cost as well as the ease of carrying out experiments, and is a major consideration for precious biological samples with limited availability. In these senses, the two solution hybrid platforms, Agilent and NimbleGen EZ, showed great advantages over the chip hybridization platform. These two solution-based platforms require smaller amounts of input DNA (approximately $3 \mu \mathrm{g}$ ) and no specialized equipment. In addition, reagent costs for these two platforms are lower when more than ten samples are being studied, and could possibly be further reduced with the introduction of sample pooling prior to the capture possess.

For performance aspects, such as the accuracy of SNP detection, GC bias and reference allele bias, and reproducibility, we did not observe great differences among the three platforms.

Taken together, our results here demonstrate that although the three platforms showed general comparability of performance, the two solution hybrid platforms would be the leading choice for most studies, especially those using large numbers of samples. In comparing these two, the Agilent platform showed a larger set of targets, targeting a more comprehensive set of human protein-coding genes and providing more complete coverage of their CDs, while the NimbleGen platform had better capture efficiency and could provide a higher proportion of CDs with high quality genotype assignments (thus higher completeness of SNP detection), and required lower sequence coverage because of its greater evenness. Thus, a choice between the two platforms is surprisingly difficult: both are highly effective and the number of targeted genes, their CD coverage, genotype sensitivity and sequencing amount/cost required must be balanced. The larger number of genes targeted by Agilent provides an overall advantage in the versions used here, but it is important to point out that both NimbleGen and Agilent are making great progress in target design. For example, in the latest (July 2011) versions, both target sets have been expanded (NimbleGen EZv.20 to $44 \mathrm{Mb}$, Agilent to $50 \mathrm{Mb}$ ), and currently cover more than $90 \%$ of annotated human genes (Table S7 in Additional file 1).

\section{Conclusions}

We demonstrate here a systematic evaluation of the performance of the current versions of three human wholeexome capture platforms. The data reported here will make it easier for researchers to more carefully assess the type of exome capture technology that will work best for their experimental goals and costs, and allow them to improve their own experimental design to take advantage or reduce the limitations of the available platform types.

\section{Materials and methods \\ Genomic DNA and kit preparation}

Genomic DNA was extracted from a lymphoblastoid cell line of YanHuang [26] using proteinase $\mathrm{K}$ and phenol/ chloroform [27] and further subjected to RNase treatment. DNA sample quality and quantity were initially characterized by gel electrophoresis and nano-drop measurement, and further quantified using the Quant-iT dsDNA HS Assay kit (0.2 to 100 ng; Invitrogen, Q32854, Carlsbad, CA, USA). NimbleGen Sequence Capture Array (Human Exome 2.1 M Array) and SeqCap EZ (v1.0) kits, and Agilent SureSelect kits (Human All Exon Kits) were purchased from their respective manufacturer.

\section{Exome capture library preparation}

Prior to library construction, we optimized the manufacturers' standard protocols for our sequencing pipeline. Major parameters for optimization included input DNA quantity, fragmentation size, number of PCR cycles and indexing system. As a result, we adopted the following protocol.

Input DNA quantities of $10 \mu \mathrm{g}, 3 \mu \mathrm{g}$ and $3 \mu \mathrm{g}$ were used for NimbleGen Sequence Capture Array, NimbleGen SeqCap EZ and Agilent SureSelect library preparation, respectively. The DNAs were fragmented to 200 to $250 \mathrm{bp}$ in size, followed by end-repair, A-tailing and BGI paired-end index adapter ligation, following the Illumina DNA library preparation protocol described elsewhere [28]. Four cycles of pre-capture amplification were then conducted with Platinum Pfx DNA polymerase (Invitrogen) under the PCR conditions: 2 minutes at $94^{\circ} \mathrm{C}$; four cycles of $10 \mathrm{~s}$ at $94^{\circ} \mathrm{C}, 30 \mathrm{~s}$ at $62^{\circ} \mathrm{C}$, and $30 \mathrm{~s}$ at $72^{\circ} \mathrm{C}$; then $300 \mathrm{~s}$ at $72^{\circ} \mathrm{C}$. $\mathrm{PCR}$ products were further analyzed using a Bioanalyzer (Agilent, Santa Clara, CA, USA), and quantified by Qubit BR measurement (Invitrogen) before performing exome capture.

Exome capture was performed with the PCR products following each of the three manufacturers' standard 
protocols. Then, ten cycles of post-capture amplification were conducted using Platinum Pfx DNA polymerase (Invitrogen) under slightly modified PCR conditions compared to pre-capture amplification $\left(2\right.$ minutes at $94^{\circ}$ C; 10 cycles of $10 \mathrm{~s}$ at $94^{\circ} \mathrm{C}, 30 \mathrm{~s}$ at $58^{\circ} \mathrm{C}$, and $30 \mathrm{~s}$ at $72^{\circ} \mathrm{C}$; then $300 \mathrm{~s}$ at $72^{\circ} \mathrm{C}$ ). Final libraries were validated by Bioanalyzer analysis (Agilent) and quantitative PCR, in preparation for massively parallel sequencing.

\section{Sequencing and pre-mapping data process}

Sequencing of each library was done on an Illumina HiSeq2000 as paired-end 90-bp reads (PE90) after indexing individual libraries and pooling them in pairs. Each library was initially sequenced to a depth providing an approximately 30 -fold mapped coverage on targeted regions, and then one of the two replicates was further chosen from each of the three platforms to sequence to $>60$-fold coverage on targeted regions. Raw image files were processed by the Illumina pipeline (version 1.3.4) for base calling and to generate a raw read set. Adapter contamination and reads of low quality (more than four ' $\mathrm{N}$ ' nucleotides) were identified and removed before mapping.

\section{Mapping, genotype and SNP calling}

SOAP (v2.21) [29] was used to align the reads to the NCBI human genome reference assembly (build 36.3) with parameters set to '-a - b -D -o - $\mathrm{u}-\mathrm{t}-135-\mathrm{n} 4-\mathrm{r} 1$ -2 -v 2 -s 40'. These settings provided the best mapping rate by giving highest priority to paired-end and then lower priority to unpaired single-end matches. SOAPsnp [30] (v1.03) was used to call consensus genotypes with the parameters ' $-\mathrm{i}-\mathrm{d}-\mathrm{o}-\mathrm{r} 0.00005-\mathrm{e} 0.0001-\mathrm{t}-\mathrm{s}-2-\mathrm{u}$ $-\mathrm{M}-\mathrm{L}-\mathrm{T}$ ', where '- $\mathrm{T}$ ' used the targeted and flanking regions. As our sample is from a male, we added the parameter '- $m$ ' for the sex chromosomes. Then SNPs were extracted from the consensus genotype file, and those with sequencing depth $<10$, quality $<30$, copy number of a nearby region $>2$, or other SNPs within 5 bp were filtered out to get high-confidence calls.

\section{Data accessibility}

All data described here are being deposited to the NCBI Sequence Read Archive [SRA:035389].

\section{Additional material}

Additional file 1: Supplementary Tables 1 to 7 .

Additional file 2: Supplementary Figures 1 to 5 .

\section{Abbreviations}

bp: base pair; CCDS: consensus coding sequences; CD: coding sequence; Gb: billion base pairs; HGMD: Human Gene Mutation Database; Mb: million base pairs; PE90: paired-end 90-bp read; SNP: single nucleotide polymorphism; WGSS: whole-genome shotgun sequencing.

\section{Acknowledgements}

We would like to acknowledge Scott Edmunds and Laurie Goodman for help with manuscript preparation; BGI-Shenzhen targeted enrichment team and BGI-Shenzhen genome sequencing platforms for assistance with exome capture, sequencing and data processing. This work was supported in part by grants from the National High Technology Research and Development Program of China (863 Program, 2006AA02A302 to HM-Y, 2009 AA022707 to $X Q-Z$ ), and National Natural Science Foundation of China (30811130531 to HM-Y). Chris Tyler-Smith and Yali Xue were supported by The Wellcome Trust.

\section{Author details}

${ }^{1}$ Beijing Genomics Institute at Shenzhen, 11F, Bei Shan Industrial Zone, Yantian District, Shenzhen 518083, China. ${ }^{2}$ Beijing Institute of Genomics, Chinese Academy of Sciences, No.7 Beitucheng West Road, Chaoyang District, Beijing 100029, China. ${ }^{3}$ Graduate University of Chinese Academy Sciences, 19A Yuquanlu, Beijing 100049, China. ${ }^{4}$ The Wellcome Trust Sanger Institute, Wellcome Trust Genome Campus, Hinxton, Cambridge CB10 1SA, UK.

\section{Authors' contributions}

XZ and HY designed the study. YuX, JiaW and TJ analyzed all data. JiW, JuW, $M W, X L, G T$ and $H J$ developed the optimized exome capture protocols, and conducted library preparation and sequencing. A designed the study, coordinated all work and wrote the manuscript. CTS designed the study and revised the manuscript. YaX revised the manuscript. All authors read and approved the final manuscript.

\section{Competing interests}

The authors declare that they have no competing interests.

Received: 23 May 2011 Revised: 20 July 2011

Accepted: 28 September 2011 Published: 28 September 2011

\section{References}

1. Lander ES, Linton LM, Birren B, Nusbaum C, Zody MC, Baldwin J, Devon K, Dewar K, Doyle M, FitzHugh W, Funke R, Gage D, Harris K, Heaford A, Howland J, Kann L, Lehoczky J, LeVine R, McEwan P, McKernan K, Meldrim J, Mesirov JP, Miranda C, Morris W, Naylor J, Raymond C, Rosetti M, Santos R, Sheridan A, Sougnez C, et al: Initial sequencing and analysis of the human genome. Nature 2001, 409:860-921.

2. Venter JC, Adams MD, Myers EW, Li PW, Mural RJ, Sutton GG, Smith HO, Yandell M, Evans CA, Holt RA, Gocayne JD, Amanatides P, Ballew RM, Huson DH, Wortman JR, Zhang Q, Kodira CD, Zheng XH, Chen L, Skupski M, Subramanian G, Thomas PD, Zhang J, Gabor Miklos GL, Nelson C, Broder S, Clark AG, Nadeau J, McKusick VA, Zinder N, et al: The sequence of the human genome. Science 2001, 291:1304-1351.

3. Antonarakis SE, Beckmann JS: Mendelian disorders deserve more attention. Nat Rev Genet 2006, 7:277-282.

4. Manolio TA, Collins FS, Cox NJ, Goldstein DB, Hindorff LA, Hunter DJ, McCarthy MI, Ramos EM, Cardon LR, Chakravarti A, Cho JH, Guttmacher AE, Kong A, Kruglyak L, Mardis E, Rotimi CN, Slatkin M, Valle D, Whittemore AS, Boehnke M, Clark AG, Eichler EE, Gibson G, Haines JL, Mackay TF, McCarroll SA, Visscher PM: Finding the missing heritability of complex diseases. Nature 2009, 461:747-753.

5. Altshuler D, Daly MJ, Lander ES: Genetic mapping in human disease. Science 2008, 322:881-888.

6. Stratton MR, Campbell PJ, Futreal PA: The cancer genome. Nature 2009, 458:719-724.

7. Margulies M, Egholm M, Altman WE, Attiya S, Bader JS, Bemben LA, Berka J, Braverman MS, Chen YJ, Chen Z, Dewell SB, Du L, Fierro JM, Gomes XV, Godwin BC, He W, Helgesen S, Ho CH, Irzyk GP, Jando SC, Alenquer ML, Jarvie TP, Jirage KB, Kim JB, Knight JR, Lanza JR, Leamon JH, Lefkowitz SM, Lei $\mathrm{M}, \mathrm{Li}$ J, et al: Genome sequencing in microfabricated high-density picolitre reactors. Nature 2005, 437:376-380.

8. Bentley DR, Balasubramanian S, Swerdlow HP, Smith GP, Milton J, Brown CG, Hall KP, Evers DJ, Barnes CL, Bignell HR, Boutell JM, Bryant J, Carter RJ, Keira Cheetham R, Cox AJ, Ellis DJ, Flatbush MR, Gormley NA, 
Humphray SJ, Irving LJ, Karbelashvili MS, Kirk SM, Li H, Liu X, Maisinger KS, Murray $L$, Obradovic B, Ost T, Parkinson ML, Pratt MR, et al: Accurate whole human genome sequencing using reversible terminator chemistry. Nature 2008, 456:53-59.

9. McKernan KJ, Peckham HE, Costa GL, McLaughlin SF, Fu Y, Tsung EF, Clouser CR, Duncan C, Ichikawa JK, Lee CC, Zhang Z, Ranade SS, Dimalanta ET, Hyland FC, Sokolsky TD, Zhang L, Sheridan A, Fu H, Hendrickson CL, Li B, Kotler L, Stuart JR, Malek JA, Manning JM, Antipova AA, Perez DS, Moore MP, Hayashibara KC, Lyons MR, Beaudoin RE, et al: Sequence and structural variation in a human genome uncovered by short-read, massively parallel ligation sequencing using two-base encoding. Genome Res 2009, 19:1527-1541.

10. Drmanac R, Sparks AB, Callow MJ, Halpern AL, Burns NL, Kermani BG, Carnevali P, Nazarenko I, Nilsen GB, Yeung G, Dahl F, Fernandez A, Staker B, Pant KP, Baccash J, Borcherding AP, Brownley A, Cedeno R, Chen L, Chernikoff D, Cheung A, Chirita R, Curson B, Ebert JC, Hacker CR, Hartlage R, Hauser $B$, Huang $S$, Jiang $Y$, Karpinchyk $V$, et al: Human genome sequencing using unchained base reads on self-assembling DNA nanoarrays. Science 2010, 327:78-81.

11. Ng SB, Turner EH, Robertson PD, Flygare SD, Bigham AW, Lee C, Shaffer T, Wong $M$, Bhattacharjee A, Eichler EE, Bamshad M, Nickerson DA, Shendure J: Targeted capture and massively parallel sequencing of 12 human exomes. Nature 2009, 461:272-276.

12. Choi M, Scholl UI, Ji W, Liu T, Tikhonova IR, Zumbo P, Nayir A, Bakkaloglu A, Ozen S, Sanjad S, Nelson-Williams C, Farhi A, Mane S, Lifton RP: Genetic diagnosis by whole exome capture and massively parallel DNA sequencing. Proc Natl Acad Sci USA 2009, 106:19096-19101.

13. Gnirke A, Melnikov A, Maguire J, Rogov P, LeProust EM, Brockman W, Fennell T, Giannoukos G, Fisher S, Russ C, Gabriel S, Jaffe DB, Lander ES, Nusbaum C: Solution hybrid selection with ultra-long oligonucleotides for massively parallel targeted sequencing. Nat Biotechnol 2009, 27:182-189.

14. Bainbridge MN, Wang M, Burgess DL, Kovar C, Rodesch MJ, D'Ascenzo M, Kitzman J, Wu YQ, Newsham I, Richmond TA, Jeddeloh JA, Muzny D, Albert TJ, Gibbs RA: Whole exome capture in solution with $3 \mathrm{Gbp}$ of data. Genome Biol 2010, 11:R62.

15. Ng SB, Buckingham K, Lee C, Bigham AW, Tabor HK, Dent KM, Huff CD, Shannon PT, Jabs EW, Nickerson DA, Shendure J, Bamshad MJ: Exome sequencing identifies the cause of a mendelian disorder. Nat Genet 2010, 42:30-35.

16. Bilguvar K, Ozturk AK, Louvi A, Kwan KY, Choi M, Tatli B, Yalnizoglu D, Tuysuz B, Caglayan AO, Gokben S, Kaymakcalan H, Barak T, Bakircioglu M, Yasuno K, Ho W, Sanders S, Zhu Y, Yilmaz S, Dincer A, Johnson MH, Bronen RA, Kocer N, Per H, Mane S, Pamir MN, Yalcinkaya C, Kumandas S, Topcu M, Ozmen M, Sestan N, et al: Whole-exome sequencing identifies recessive WDR62 mutations in severe brain malformations. Nature 2010, 467:207-210

17. Walsh $T$, Shahin H, Elkan-Miller T, Lee MK, Thornton AM, Roeb W, Abu Rayyan A, Loulus S, Avraham KB, King MC, Kanaan M: Whole exome sequencing and homozygosity mapping identify mutation in the cell polarity protein GPSM2 as the cause of nonsyndromic hearing loss DFNB82. Am J Hum Genet 2010, 87:90-94.

18. Haack TB, Danhauser K, Haberberger B, Hoser J, Strecker V, Boehm D, Uziel G, Lamantea E, Invernizzi F, Poulton J, Rolinski B, luso A, Biskup S, Schmidt T, Mewes HW, Wittig I, Meitinger T, Zeviani M, Prokisch H: Exome sequencing identifies ACAD9 mutations as a cause of complex I deficiency. Nat Genet 2010, 42:1131-1134.

19. Ng SB, Bigham AW, Buckingham KJ, Hannibal MC, McMillin MJ, Gildersleeve HI, Beck AE, Tabor HK, Cooper GM, Mefford HC, Lee C, Turner EH, Smith JD, Rieder MJ, Yoshiura K, Matsumoto N, Ohta T, Niikawa N, Nickerson DA, Bamshad MJ, Shendure J: Exome sequencing identifies MLL2 mutations as a cause of Kabuki syndrome. Nat Genet 2010, 42:790-793.

20. Musunuru K, Pirruccello JP, Do R, Peloso GM, Guiducci C, Sougnez C, Garimella KV, Fisher S, Abreu J, Barry AJ, Fennell T, Banks E, Ambrogio L, Cibulskis K, Kernytsky A, Gonzalez E, Rudzicz N, Engert JC, DePristo MA, Daly MJ, Cohen JC, Hobbs HH, Altshuler D, Schonfeld G, Gabriel SB, Yue P, Kathiresan S: Exome sequencing, ANGPTL3 mutations, and familial combined hypolipidemia. N Engl J Med 2010, 363:2220-2227.

21. Wang JL, Yang $X$, Xia K, Hu ZM, Weng L, Jin X, Jiang H, Zhang $P$, Shen $L$, Guo JF, Li N, Li YR, Lei LF, Zhou J, Du J, Zhou YF, Pan Q, Wang J, Li RQ,
Tang BS: TGM6 identified as a novel causative gene of spinocerebellar ataxias using exome sequencing. Brain 2010, 133:3510-3518.

22. Hodges E, Xuan Z, Balija V, Kramer M, Molla MN, Smith SW, Middle CM, Rodesch MJ, Albert TJ, Hannon GJ, McCombie WR: Genome-wide in situ exon capture for selective resequencing. Nat Genet 2007, 39:1522-1527.

23. Mamanova L, Coffey AJ, Scott CE, Kozarewa I, Turner EH, Kumar A, Howard E, Shendure J, Turner DJ: Target-enrichment strategies for nextgeneration sequencing. Nat Methods 2010, 7:111-118.

24. Turner EH, Ng SB, Nickerson DA, Shendure J: Methods for genomic partitioning. Annu Rev Genomics Hum Genet 2009, 10:263-284.

25. Teer JK, Bonnycastle LL, Chines PS, Hansen NF, Aoyama N, Swift AJ, Abaan HO, Albert TJ, Margulies EH, Green ED, Collins FS, Mullikin JC, Biesecker LG: Systematic comparison of three genomic enrichment methods for massively parallel DNA sequencing. Genome Res 2010, 20:1420-1431.

26. Wang J, Wang W, Li R, Li Y, Tian G, Goodman L, Fan W, Zhang J, Li J, Guo Y, Feng B, Li H, Lu Y, Fang X, Liang H, Du Z, Li D, Zhao Y, Hu Y, Yang Z, Zheng H, Hellmann I, Inouye M, Pool J, Yi X, Zhao J, Duan J, Zhou Y, Qin J, Ma L, et al: The diploid genome sequence of an Asian individual. Nature 2008, 456:60-65.

27. Blin N, Stafford DW: A general method for isolation of high molecular weight DNA from eukaryotes. Nucleic Acids Res 1976, 3:2303-2308.

28. Quail MA, Kozarewa I, Smith F, Scally A, Stephens PJ, Durbin R, Swerdlow H, Turner DJ: A large genome center's improvements to the Illumina sequencing system. Nat Methods 2008, 5:1005-1010.

29. Li R, Yu C, Li Y, Lam TW, Yiu SM, Kristiansen K, Wang J: SOAP2: an improved ultrafast tool for short read alignment. Bioinformatics 2009, 25:1966-1967.

30. Li R, Li Y, Fang X, Yang H, Wang J, Kristiansen K: SNP detection for massively parallel whole-genome resequencing. Genome Res 2009 19:1124-1132

doi:10.1186/gb-2011-12-9-r95

Cite this article as: Asan et al:: Comprehensive comparison of three commercial human whole-exome capture platforms. Genome Biology 2011 12:R95.

\section{Submit your next manuscript to BioMed Central and take full advantage of:}

- Convenient online submission

- Thorough peer review

- No space constraints or color figure charges

- Immediate publication on acceptance

- Inclusion in PubMed, CAS, Scopus and Google Scholar

- Research which is freely available for redistribution

Submit your manuscript at www.biomedcentral.com/submit
C) Biomed Central 